\title{
A chiral $N=1$ type I vacuum in four dimensions and its heterotic dual
}

\author{
Zurab Kakushadze* \\ Lyman Laboratory of Physics, Harvard University, Cambridge, Massachusetts 02138 \\ and Department of Physics, Northeastern University, Boston, Massachusetts 02115 \\ Gary Shiu ${ }^{\dagger}$ \\ Newman Laboratory of Nuclear Studies, Cornell University, Ithaca, New York 14853-5001
}

(Received 21 May 1997)

\begin{abstract}
In this paper we consider type I string theory compactified on a $\mathbf{Z}_{7}$ orbifold. The model has $N=1$ supersymmetry, a $\mathrm{U}(4) \otimes \mathrm{U}(4) \otimes \mathrm{U}(4) \otimes \mathrm{SO}(8)$ gauge group, and chiral matter. There are only $D 9$-branes (for which we discuss tadpole cancellation conditions) in this model corresponding to a perturbative heterotic description in a certain region of the moduli space. We construct the heterotic dual, match the perturbative type I and heterotic tree-level massless spectra via giving certain scalars appropriate vacuum expectation values, VEVs, and point out the crucial role of the perturbative superpotential (on the heterotic side) for this matching. The relevant couplings in this superpotential turn out to be nonrenormalizable (unlike the $Z$-orbifold case discussed by Kakushadze, where Yukawa couplings sufficed for duality matching). We also discuss the role of the anomalous $\mathrm{U}(1)$ gauge symmetry present in both type I and heterotic models. In the perturbative regime we match the (tree-level) moduli spaces of these models. We point out possible generalizations of the $\mathbf{Z}_{3}$ and $\mathbf{Z}_{7}$ cases to include $D 5$-branes which would help in understanding nonperturbative five-brane dynamics on the heterotic side. [S0556-2821(97)03618-7]
\end{abstract}

PACS number(s): $11.25 . \mathrm{Mj}$

\section{INTRODUCTION}

In recent years nonperturbative string dynamics has been coming under greater control. String dualities have been playing an important role in this process, as they allow us to address nonperturbative issues in a given string theory by studying them perturbatively in a dual theory. Supersymmetry has been a key ingredient of string duality, as the larger the number of unbroken space-time supersymmetries, the better handle we have over nonperturbative string dynamics. Thus, much progress has been made in understanding $N=4$ and $N=2$ string dualities, and now the attention is shifting toward grasping $N=1$ cases.

$N=1$ type I-heterotic duality in four dimensions is a promising arena for testing the validity of the idea of $N=1$ string dualities, as well as for developing tools that might help understand nonperturbative effects in, say, heterotic string theory (e.g., dynamics of five-branes responsible for enhanced gauge symmetries). The tree-level relation between type I and heterotic dilatons in $D$ space-time dimensions [2] (which follows from the conjectured type I-heterotic duality in ten dimensions [3]) reads

$$
\phi_{H}=\frac{6-D}{4} \phi_{I}-\frac{D-2}{16} \ln \left[\operatorname{det}\left(g_{I}\right)\right] .
$$

Here $g_{I}$ is the internal metric of the type I compactification space, whereas $\phi_{I}$ and $\phi_{H}$ are the type I and heterotic dilatons, respectively. One implication of the above equation is that in four dimensions there always exists a region in the

\footnotetext{
*Electronic address: zurab@string.harvard.edu

†Electronic address: shiu@mail.lns.cornell.edu
}

moduli space where both type I and heterotic string theories are weekly coupled, and there we can rely on perturbation theory. If we understand the map between perturbative effects in the two descriptions, we may be able to learn about nonperturbative effects in, say, heterotic string via casting them into perturbative effects in type I theory (e.g., nonperturbative dynamics of heterotic five-branes can presumably be understood by studying perturbative dynamics of type I D5-branes).

Recently one of us studied an example of a fourdimensional $N=1$ type I-heterotic dual pair [1]. The type I model, as well as the candidate heterotic dual, considered in Ref. [1] were first constructed in Ref. [2]. The type I model is a compactification on the $Z$ orbifold (and has $D 9$-branes only), whereas the candidate heterotic dual is a $Z$-orbifold compactification with a nonstandard embedding of the gauge connection. At the orbifold points the tree-level massless spectra of the two models differ as there are extra twisted matter fields in the heterotic model that do not have (perturbative) type I counterparts. As discussed in Ref [1], there is a tree-level superpotential in the heterotic model precisely such that the extra states become heavy after appropriate use of the Higgs mechanism. The role of the anomalous U(1) (present in both type I and heterotic models) was also discussed in Ref. [1].

The case studied in Ref. [1] is remarkable in the sense that the type I model has only $D 9$-branes and the dynamics is completely perturbative from the heterotic point of view, hence there is not much difficulty in establishing (tree-level) duality. In the context of our previous discussion, it would be important to see if there is any pattern in such perturbative $N=1$ type I-heterotic duality in four dimensions. If so, this would help separate perturbative effects from nonperturbative ones in the cases with $D 5$-branes (which are more in- 
volved from the heterotic point of view, and these are the cases we would ultimately like to understand). There turns out to be one other case of type I $\mathbf{Z}_{N}$ orbifold compactification with $N=1$ supersymmetry and no $D 5$-branes. This is the compactification on the $\mathbf{Z}_{7}$ orbifold that we study in this paper.

Before discussing the $\mathbf{Z}_{7}$ case, we list some orbifolds of type I strings with $N=1$ supersymmetry in four dimensions (4D) (there are two inequivalent $\mathbf{Z}_{6}$ orbifolds in $4 \mathrm{D}$ ): $D$ 9-branes only: (i) $\mathbf{Z}_{3}$, (ii) $\mathbf{Z}_{7} ; D 9$-branes and $D 5$-branes: (iii) $\mathbf{Z}_{6}$, (iv) $\mathbf{Z}_{6}^{\prime}$, (v) $\mathbf{Z}_{2} \otimes \mathbf{Z}_{2}$, (vi) $\mathbf{Z}_{4}$. So far, only the $\mathbf{Z}_{3}$ [2] and $\mathbf{Z}_{2} \otimes \mathbf{Z}_{2}$ [4] cases have been constructed, and only the $\mathbf{Z}_{3}$ case has been studied from the type I-heterotic duality point of view. In this paper we discuss the $\mathbf{Z}_{7}$ case. The model has $N=1 \quad$ supersymmetry, $\mathrm{U}(4) \otimes \mathrm{U}(4) \otimes \mathrm{U}(4) \otimes \mathrm{SO}(8)$ gauge group, and chiral matter. There is anomalous $\mathrm{U}(1)$ in this model. We also construct the heterotic dual that has the same gauge symmetry and matter content as the type I model except for extra twisted matter fields. This is just as in the $\mathbf{Z}_{3}$ model, albeit there are some subtle differences in the way these extra matter fields are charged under the gauge group. Another difference between the $\mathbf{Z}_{3}$ and $\mathbf{Z}_{7}$ cases is that the orbifold blow-up modes in the former case are charged under the anomalous $\mathrm{U}(1)$ and contribute to cancelling the $D$-term, whereas in the latter case the orbifold blow-up modes are neutral under the anomalous $\mathrm{U}(1)$. This results in different pictures for embedding of the type I moduli space into that of heterotic string in the $\mathbf{Z}_{3}$ and $\mathbf{Z}_{7}$ cases. Just as in the $\mathbf{Z}_{3}$ case, in the $\mathbf{Z}_{7}$ case there is a tree-level superpotential that after appropriate use of the Higgs mechanism gives masses to all the extra twisted matter in the heterotic model, and the massless spectra of the type I and heterotic strings are matched. There is also a difference, however: in the $\mathbf{Z}_{3}$ case renormalizable (Yukawa) couplings are sufficient for duality matching, whereas in the $\mathbf{Z}_{7}$ case the corresponding couplings are nonrenormalizable. (In deducing the heterotic superpotential the tools developed in Ref. [5] prove to be very useful; see Appendix B and Appendix C for details.) With these subtle differences, the type I-heterotic duality in the two cases $\left(\mathbf{Z}_{3}\right.$ and $\mathbf{Z}_{7}$ ) works much in the same way, and there is, henceforth, a clear pattern we see from studying these examples. (Note that perturbative superpotentials also seem to be necessary for matching the massless spectra of $F$ theory and heterotic dual pairs [6].)

The paper is organized as follows. In Sec. II we discuss the $\mathbf{Z}_{7}$ orbifold type I model. In Sec. III we construct the heterotic dual. In Sec. IV we give perturbative superpotentials for these models. In Sec. V we discuss the moduli space, and explain the matching between the type I and heterotic moduli spaces, as well as their tree-level spectra. In Sec. VI we give conclusions and remarks. Some of the details regarding the tadpole cancellation in type I theory, and also the heterotic superpotential are relegated to the Appendixes.

\section{TYPE I MODEL}

In this section we discuss the construction of the type I model. Let us start from the type IIB string model compactified on the six-torus which has a $\mathbf{Z}_{7}$ rotational symmetry. [A more detailed discussion of this six-torus will be given in the next section where we go through the construction of the (candidate) heterotic dual of the model considered in this section.] This model has $N=8$ supersymmetry. Let us now consider the symmetric $\mathbf{Z}_{7}$ orbifold model generated by the twist

$$
T_{7}=\left(\theta, \theta^{2}, \theta^{3} \| \theta, \theta^{2}, \theta^{3}\right) .
$$

Here $\theta$ is a $2 \pi / 7$ rotation of a complex boson (we have complexified the six real bosons into three complex bosons). The double vertical line separates the right and left movers of the string. The resulting model has $N=2$ space-time supersymmetry. This model has the following moduli. There are 8 Neveu-Schwarz-Neveu-Schwarz (NS-NS) fields $\phi, B_{\mu \nu}, B_{i \bar{i}}, g_{i} \bar{i}$, and 8 Ramond-Ramond (R-R) fields $\phi^{\prime}, B_{\mu \nu}^{\prime}, B_{i \bar{i}}^{\prime}, C_{\mu \nu i \bar{i}}^{\prime}$.

Let us now consider the orientifold projection of this model. The closed string sector (which is simply the subspace of the Hilbert space of the original type IIB spectrum invariant under the orientifold projection $\Omega$ ) contains the $N=1$ supergravity multiplet, and 3 untwisted (the NS-NS fields that survive the $\Omega$ projection are $g_{i} \bar{i}$, whereas the R-R fields that are kept are $B_{i \bar{i}}^{\prime}$; note that the NS-NS field $\phi$ and the R-R field $B_{\mu \nu}^{\prime}$ also survive and enter in the dilaton supermultiplet) and 21 twisted chiral supermultiplets (which are neutral under the gauge group of the model). For consistency (tadpole cancellation; see Appendix A for details) we must include the open string sector. Note that in this model we only have $D 9$-branes but no $D 5$-branes since the orbifold group does not contain an order two element. (If the orbifold group contains an order two element $R$, then the sector $R \Omega$ would contain $D 5$-branes). Thus, we only have 99 open strings. The gauge group consistent with tadpole cancellation then is $\mathrm{U}(4) \otimes \mathrm{U}(4) \otimes \mathrm{U}(4) \otimes \mathrm{SO}(8)$. The 99 open strings also give rise to the chiral matter fields $\quad\left(\mathbf{4}, \mathbf{1}, \mathbf{1}, \mathbf{8}_{v}\right)(+1,0,0)_{L}, \quad(\overline{\mathbf{4}}, \overline{\mathbf{4}}, \mathbf{1}, \mathbf{1})(-1,-1,0)_{L}$, $(\overline{\mathbf{4}}, \mathbf{4}, \mathbf{1}, \mathbf{1})(-1,+1,0)_{L}$, and $(\mathbf{6}, \mathbf{1}, \mathbf{1}, \mathbf{1})(+2,0,0)_{L}$. In addition, there are fields that can be obtained by permuting the three U(4)'s [this permutation must be accompanied by changing the irreducible representation (irrep) of the third U(4) to its complex conjugate]. Here the first four entries in bold font indicate the irreps of the $\mathrm{SU}(4) \otimes \mathrm{SU}(4) \otimes \mathrm{SU}(4) \otimes \mathrm{SO}(8) \quad$ subgroup, $\quad$ whereas the $\mathrm{U}(1)^{3}$ charges are given in the parenthesis. The subscript $L$ indicates the space-time helicity of the corresponding fermionic fields. The massless spectrum of this model is summarized in Table I.

Note that the $\mathrm{U}(1)^{3}$ gauge symmetry is anomalous. We can form a linear combination of these U(1)'s such that only one of them is anomalous [this combination is given by $Q_{1}+Q_{2}-Q_{3}$, where $Q_{1,2,3}$ are the first, second, and third $\mathrm{U}(1)$ charges, respectively]. The total $\mathrm{U}(1)$ anomaly is +36 . By the generalized Green-Schwarz mechanism $[7,8]$ some of the fields charged under $\mathrm{U}(1)$ will acquire vacuum expectation values (VEV's) to cancel the Fayet-Illiopoulos $D$-term.

\section{HETEROTIC STRING MODEL}

In this section we give the construction of the heterotic string model that is (candidate) dual to the type I model considered in the previous section. Let us start from the 
TABLE I. The massless spectrum of the type I model with $N=1 \quad$ space-time supersymmetry and gauge group $\mathrm{SU}(4) \otimes \mathrm{SU}(4) \otimes \mathrm{SU}(4) \otimes \mathrm{SO}(8) \otimes \mathrm{U}(1)^{3}$ discussed in Sec. II. The gravity, dilaton and gauge supermultiplets are not shown.

\begin{tabular}{lccc}
\hline \hline & & $\mathrm{SU}(4) \otimes \mathrm{SU}(4)$ & \\
Sector & Field & $\otimes \mathrm{SU}(4) \otimes \mathrm{SO}(8) \otimes \mathrm{U}(1)^{3}$ & Comments \\
\hline Closed & & & \\
Untwisted & $\phi_{a}$ & $3(\mathbf{1}, \mathbf{1}, \mathbf{1}, \mathbf{1})(0,0,0)_{L}$ & $a=1,2,3$ \\
Closed & $S_{\alpha}^{a}$ & $21(\mathbf{1}, \mathbf{1}, \mathbf{1}, \mathbf{1})(0,0,0)_{L}$ & $a=1$ to 3 \\
Twisted & & & $\alpha=1$ to 7 \\
& $P_{1}$ & $\left(\mathbf{4}, \mathbf{1}, \mathbf{1}, \mathbf{8}_{v}\right)(+1,0,0)_{L}$ & \\
& $P_{2}$ & $\left(\mathbf{1}, \mathbf{4}, \mathbf{1}, \mathbf{8}_{v}\right)(0,+1,0)_{L}$ & \\
& $P_{3}$ & $\left(\mathbf{1}, \mathbf{1}, \overline{\mathbf{4}}, \mathbf{8}_{v}\right)(0,0,-1)_{L}$ & \\
& $Q_{1}$ & $(\mathbf{1}, \overline{\mathbf{4}}, \mathbf{4}, \mathbf{1})(0,-1,+1)_{L}$ & \\
& $Q_{2}$ & $(\overline{\mathbf{4}}, \mathbf{1}, \mathbf{4}, \mathbf{1})(-1,0,+1)_{L}$ & \\
Open & $Q_{3}$ & $(\overline{\mathbf{4}}, \overline{\mathbf{4}}, \mathbf{1}, \mathbf{1})(-1,-1,0)_{L}$ & \\
& $R_{1}$ & $(\overline{\mathbf{4}}, \mathbf{4}, \mathbf{1}, \mathbf{1})(-1,+1,0)_{L}$ & \\
& $R_{2}$ & $(\mathbf{1}, \overline{\mathbf{4}}, \mathbf{4}, \mathbf{1})(0,-1,-1)_{L}$ & \\
& $R_{3}$ & $(\mathbf{4 , 1}, \mathbf{4}, \mathbf{1})(+1,0,+1)_{L}$ & \\
$\Phi_{1}$ & $(\mathbf{1}, \mathbf{1}, \mathbf{6}, \mathbf{1})(0,0,-2)_{L}$ & \\
$\Phi_{2}$ & $(\mathbf{6 , 1}, \mathbf{1}, \mathbf{1})(+2,0,0)_{L}$ & \\
$\Phi_{3}$ & $(\mathbf{1}, \mathbf{6}, \mathbf{1}, \mathbf{1})(0,+2,0)_{L}$ & \\
\hline \hline
\end{tabular}

Narain model with $N=4$ space-time supersymmetry in four dimensions. Let the momenta of the internal (6 right-moving and 22 left-moving) world-sheet bosons span the (even selfdual) Narain lattice $\Gamma^{6,22}=\Gamma^{6,6} \otimes \Gamma^{16}$. Here $\Gamma^{16}$ is the $\operatorname{spin}(32) / \mathbf{Z}_{2}$ lattice, whereas the lattice $\Gamma^{6,6}$ is spanned by the momenta $\left(p_{R} \| p_{L}\right)$ with

$$
p_{L, R}=\frac{1}{2} m_{i} \widetilde{e}^{i} \pm n^{i} e_{i} .
$$

Here $m_{i}$ and $n^{i}$ are integers, $e_{i} \cdot e_{j}=g_{i j}$ is the constant background metric of the compactification manifold (six-torus), and $e_{i} \cdot \widetilde{e^{j}}=\delta_{i}{ }^{j}$. Note that we could have included the constant antisymmetric background tensor field $B_{i j}$, but for now we will set it equal to zero for the reasons that will become clear in the following (see Appendix A for details).

This Narain model has the gauge group $\mathrm{SO}(32) \otimes \mathrm{U}(1)^{6}$. The first factor $\mathrm{SO}(32)$ comes from the $\Gamma^{16}$ lattice (the 480 roots of length squared 2), and 16 oscillator excitations of the corresponding world-sheet bosons [the latter being in the Cartan subalgebra of $\mathrm{SO}(32)]$. The factor $\mathrm{U}(1)^{6}$ comes from the oscillator excitations of the six left-moving world-sheet bosons corresponding to $\Gamma^{6,6}$. Note that there are also six additional vector bosons coming from the oscillator excitations of the right-moving world-sheet bosons corresponding to $\Gamma^{6,6}$. These vector bosons are part of the $N=4$ supergravity multiplet.

Next consider the $\mathbf{Z}_{7}$ orbifold model (with nonstandard embedding of the gauge connection) obtained via twisting the above Narain model by the following $\mathbf{Z}_{7}$ twist:

$$
T_{7}=\left[\theta, \theta^{2}, \theta^{3}|| \theta, \theta^{2}, \theta^{3} \mid\left(\frac{1}{7}\right)^{4}\left(\frac{2}{7}\right)^{4}\left(\frac{3}{7}\right)^{4} 0^{4}\right] .
$$

Here $\theta$ is a $2 \pi / 7$ rotation of a complex boson (we have complexified the original six real bosons into three complex ones). Thus, the first three entries correspond to the $\mathbf{Z}_{7}$ twists of the three right-moving complex bosons (coming from the six-torus). The double vertical line separates the right and left movers. The first three left-moving entries correspond to the $\mathbf{Z}_{7}$ twists of the three left-moving complex bosons (coming from the six-torus). The single vertical line separates the latter from the sixteen real bosons corresponding to the $\Gamma^{16}$ lattice. The latter are written in the $\mathrm{SO}(32)$ basis. Thus, for example, $\left(+1,-1,0^{14}\right)$ is a root of $\mathrm{SO}(32)$ with length squared 2. There are 480 roots similar to this in the $\Gamma^{16}$ lattice, and they are descendents of the identity irrep of $\mathrm{SO}(32)$. The lattice $\Gamma^{16}$ also contains one of the spinor irreps as well. Thus, we will choose this spinor irrep to contain the momentum states of the form $\left( \pm \frac{1}{2}, \ldots, \pm \frac{1}{2}\right)$ with even number of plus signs.

Note that for the above twist to be a symmetry of the model it is necessary (and sufficient) that the twist acting on the $\Gamma^{6,6}$ lattice is a rotation in this lattice. This requirement constrains the possible values of the metric tensor $g_{i j}$.

Now we are ready to discuss the orbifold model generated by the above twist $T_{7}$. This model has $N=1$ space-time supersymmetry, and gauge group $\mathrm{U}(4) \otimes \mathrm{U}(4) \otimes \mathrm{U}(4) \otimes \mathrm{SO}(8)$, the same as the type I model discussed in the previous section. The untwisted sector gives rise to the $N=1$ supergravity multiplet coupled to the $N=1$ Yang-Mills gauge multiplet in the adjoint of $\mathrm{U}(4) \otimes \mathrm{U}(4) \otimes \mathrm{U}(4) \otimes \mathrm{SO}(8)$. The matter fields in the untwisted sector are the same as those in the open string sector of the type I model. There are also chiral multiplets neutral under the gauge group: $3(\mathbf{1}, \mathbf{1}, \mathbf{1}, \mathbf{1})(0,0,0)_{L}$. Note that these contain six scalar fields that are the leftover geometric moduli whose VEV's parametrize the moduli space $[\mathrm{SU}(1,1, \mathbf{Z}) \backslash \mathrm{SU}(1,1) / \mathrm{U}(1)]^{3}$. [This is the subspace of the original Narain moduli space $\mathrm{SO}(6,6, \mathbf{Z}) \backslash \mathrm{SO}(6,6) / \mathrm{SO}(6) \otimes \mathrm{SO}(6)$ that is invariant under the twist.] Actually, the (perturbative) moduli space of this model is larger, and we will return to this point later on.

Next, consider the twisted sector. In the twisted sector we have the chiral supermultiplets $7(\mathbf{1}, \mathbf{1}, \mathbf{1}, \mathbf{1})(4 / 7,8 / 7,-12 / 7)_{L}$ and $7(\mathbf{1}, \mathbf{1}, \mathbf{6}, \mathbf{1})(4 / 7,8 / 7,2 / 7)_{L}$ together with fields obtained by permuting the three U(4)'s [this permutation must be accompanied by changing the irrep of the third U(4) to its complex conjugate]. Here we note that the factor 7 comes from the number of fixed points of the $\mathbf{Z}_{7}$ orbifold we are considering.

We summarize the massless spectrum of this heterotic string model in Table II. Note that the $U(1)^{3}$ gauge symmetry is anomalous. Again, only one linear combination of the three U(1)'s is anomalous. Thus, the contributions of the untwisted and twisted sectors into the trace anomaly are +36 and $7 \times(+36)$, respectively, so that the total trace anomaly is +288 . By the generalized Green-Schwarz mechanism [7] some of the fields charged under U(1) will acquire VEV's to cancel the Fayet-Illiopoulos $D$-term.

\section{SUPERPOTENTIAL}

In this section we discuss the perturbative superpotentials for the type I and heterotic string models discussed in the previous sections. Studying the couplings and flat directions 
TABLE II. The massless spectrum of the heterotic model with $N=1$ space-time supersymmetry and gauge group $\mathrm{SU}(4) \otimes \mathrm{SU}(4) \otimes \mathrm{SU}(4) \otimes \mathrm{SO}(8) \otimes \mathrm{U}(1)^{3}$ discussed in Sec. III. The $H$ charges in both the -1 picture and the $-1 / 2$ picture are also given. The gravity, dilaton, and gauge supermultiplets are not shown.

\begin{tabular}{|c|c|c|c|c|}
\hline Sector & Field & $\mathrm{SU}(4)^{3} \otimes \mathrm{SO}(8) \otimes \mathrm{U}(1)^{3}$ & $\left(H_{1}, H_{2}, H_{3}\right)_{-1}$ & $\left(H_{1}, H_{2}, H_{3}\right)_{-1 / 2}$ \\
\hline \multirow{15}{*}{ Untwisted } & $\phi_{1}$ & $(\mathbf{1}, \mathbf{1}, \mathbf{1}, \mathbf{1})(0,0,0)_{L}$ & $(-1,0,0)$ & $\left(-\frac{1}{2},+\frac{1}{2},-\frac{1}{2}\right)$ \\
\hline & $\phi_{2}$ & $(\mathbf{1}, \mathbf{1}, \mathbf{1}, \mathbf{1})(0,0,0)_{L}$ & $(0,-1,0)$ & $\left(+\frac{1}{2},-\frac{1}{2},-\frac{1}{2}\right)$ \\
\hline & $\phi_{3}$ & $(\mathbf{1}, \mathbf{1}, \mathbf{1}, \mathbf{1})(0,0,0)_{L}$ & $(0,0,+1)$ & $\left(+\frac{1}{2},+\frac{1}{2},+\frac{1}{2}\right)$ \\
\hline & $P_{1}$ & $\left(\mathbf{4}, \mathbf{1}, \mathbf{1}, \mathbf{8}_{v}\right)(+1,0,0)_{L}$ & $(-1,0,0)$ & $\left(-\frac{1}{2},+\frac{1}{2},-\frac{1}{2}\right)$ \\
\hline & $P_{2}$ & $\left(\mathbf{1}, \mathbf{4}, \mathbf{1}, \mathbf{8}_{v}\right)(0,+1,0)_{L}$ & $(0,-1,0)$ & $\left(+\frac{1}{2},-\frac{1}{2},-\frac{1}{2}\right)$ \\
\hline & $P_{3}$ & $\left(\mathbf{1}, \mathbf{1}, \overline{\mathbf{4}}, \mathbf{8}_{v}\right)(0,0,-1)_{L}$ & $(0,0,+1)$ & $\left(+\frac{1}{2},+\frac{1}{2},+\frac{1}{2}\right)$ \\
\hline & $Q_{1}$ & $(\mathbf{1}, \overline{\mathbf{4}}, \mathbf{4}, \mathbf{1})(0,-1,+1)_{L}$ & $(-1,0,0)$ & $\left(-,+\frac{1}{2},-\frac{1}{2}\right)$ \\
\hline & $Q_{2}$ & $(\overline{\mathbf{4}}, \mathbf{1}, \mathbf{4}, \mathbf{1})(-1,0,+1)_{L}$ & $(0,-1,0)$ & $\left(+\frac{1}{2},-\frac{1}{2},-\frac{1}{2}\right)$ \\
\hline & $Q_{3}$ & $(\overline{\mathbf{4}}, \overline{\mathbf{4}}, \mathbf{1}, \mathbf{1})(-1,-1,0)_{L}$ & $(0,0,+1)$ & $\left(+\frac{1}{2},+\frac{1}{2},+\frac{1}{2}\right)$ \\
\hline & $R_{1}$ & $(\overline{\mathbf{4}}, \mathbf{4}, \mathbf{1}, \mathbf{1})(-1,+1,0)_{L}$ & $(-1,0,0)$ & $\left(-\frac{1}{2},+\frac{1}{2},-\frac{1}{2}\right)$ \\
\hline & $R_{2}$ & $(\mathbf{1}, \overline{\mathbf{4}}, \overline{\mathbf{4}}, \mathbf{1})(0,-1,-1)_{L}$ & $(0,-1,0)$ & $\left(+\frac{1}{2},-\frac{1}{2},-\frac{1}{2}\right)$ \\
\hline & $R_{3}$ & $(\mathbf{4}, \mathbf{1}, \mathbf{4}, \mathbf{1})(+1,0,+1)_{L}$ & $(0,0,+1)$ & $\left(+\frac{1}{2},+\frac{1}{2},+\frac{1}{2}\right)$ \\
\hline & $\Phi_{1}$ & $(\mathbf{1}, \mathbf{1}, \mathbf{6}, \mathbf{1})(0,0,-2)_{L}$ & $(-1,0,0)$ & $\left(-\frac{1}{2},+\frac{1}{2},-\frac{1}{2}\right)$ \\
\hline & $\Phi_{2}$ & $(\mathbf{6}, \mathbf{1}, \mathbf{1}, \mathbf{1})(+2,0,0)_{L}$ & $(0,-1,0)$ & $\left(+\frac{1}{2},-\frac{1}{2},-\frac{1}{2}\right)$ \\
\hline & $\Phi_{3}$ & $(\mathbf{1}, \mathbf{6}, \mathbf{1}, \mathbf{1})(0,+2,0)_{L}$ & $(0,0,+1)$ & $\left(+\frac{1}{2},+\frac{1}{2},+\frac{1}{2}\right)$ \\
\hline Twisted & $S_{\alpha}^{1}$ & $7(\mathbf{1}, \mathbf{1}, \mathbf{1}, \mathbf{1})(4 / 7,8 / 7,12 / 7)_{L}$ & $\left(-\frac{1}{7},-\frac{2}{7},+\frac{4}{7}\right)$ & $\left(+\frac{5}{14},+\frac{3}{14},+\frac{1}{14}\right)$ \\
\hline$\theta, \theta^{6}$ & $T_{\alpha}^{1}$ & $7(\mathbf{1}, \mathbf{1}, \mathbf{6}, \mathbf{1})(4 / 7,8 / 7,-2 / 7)_{L}$ & $\left(-\frac{1}{7},-\frac{2}{7},+\frac{4}{7}\right)$ & $\left(+\frac{5}{14},+\frac{3}{14},+\frac{1}{14}\right)$ \\
\hline Twisted & $S_{\alpha}^{2}$ & $7(\mathbf{1}, \mathbf{1}, \mathbf{1}, \mathbf{1})(8 / 7,-12 / 7,-4 / 7)_{L}$ & $\left(-\frac{2}{7},-\frac{4}{7},+\frac{1}{7}\right)$ & $\left(+\frac{3}{14},-\frac{1}{14}-\frac{5}{14}\right)$ \\
\hline$\theta^{2}, \theta^{5}$ & $T_{\alpha}^{2}$ & $7(\mathbf{1}, \mathbf{6}, \mathbf{1}, \mathbf{1})(8 / 7,2 / 7,-4 / 7)_{L}$ & $\left(-\frac{2}{7},-\frac{4}{7},+\frac{1}{7}\right)$ & $\left(+\frac{3}{14},-\frac{1}{14},-\frac{5}{14}\right)$ \\
\hline Twisted & $S_{\alpha}^{3}$ & $7(\mathbf{1}, \mathbf{1}, \mathbf{1}, \mathbf{1})(-12 / 7,4 / 7,-8 / 7)_{L}$ & $\left(-\frac{4}{7},-\frac{1}{7},+\frac{2}{7}\right)$ & $\left(-\frac{1}{14},+\frac{5}{14},-\frac{3}{14}\right)$ \\
\hline$\theta^{3}, \theta^{4}$ & $T_{\alpha}^{3}$ & $7(\mathbf{6}, \mathbf{1}, \mathbf{1}, \mathbf{1})(2 / 7,4 / 7,-8 / 7)_{L}$ & $\left(-\frac{4}{7},-\frac{1}{7},+\frac{2}{7}\right)$ & $\left(-\frac{1}{14},+\frac{5}{14},-\frac{3}{14}\right)$ \\
\hline
\end{tabular}

in these superpotentials will enable us to make the type Iheterotic duality map more precise.

Let us start from the type I model of Sec. II. We refer the reader to Table I for the massless spectum as well as our notation. Note that perturbatively the 24 chiral singlets coming from the closed string sector are flat. This can be explicitly seen by computing the scattering amplitudes for these modes within the framework of the conformal field theory of orbifolds [9]. On the other hand, the matter fields coming from the 99 open string sector have three (and, of course, some higher) point couplings. The lowest order superpotential can be written as (the calculation of the type I superpotential is completely analogous to that of the heterotic one in the untwisted sector)

$$
\begin{aligned}
W_{I}= & \lambda_{1} \epsilon_{a b c} \operatorname{Tr}\left(P_{a} P_{b} Q_{c}\right)+\lambda_{2} \operatorname{Tr}\left(Q_{1} R_{2} \Phi_{3}+Q_{2} R_{3} \Phi_{1}\right. \\
& \left.+Q_{3} R_{1} \Phi_{2}\right)+\lambda_{3} \operatorname{Tr}\left(R_{1} R_{2} R_{3}\right)+\cdots
\end{aligned}
$$

Due to the presence of the anomalous U(1), some of the fields that are charged under this U(1) (namely, $Q_{a}$ ) must acquire VEV's to cancel the Fayet-Illiopoulos $D$-term. This results in breakdown of gauge symmetry, yet the space-time supersymmetry is preserved.

Now let us turn to the heterotic string model. The superpotential of this model is more involved than that of the type I model as there are nontrivial couplings between the untwisted and the twisted sector fields. We refer the reader to
Appendix B and Appendix C for the details of calculating these couplings. The superpotential for the heterotic string model thus reads (here we are only interested in the general structure of the nonvanishing terms):

$$
\begin{aligned}
W_{H}= & \lambda_{1}^{\prime} \epsilon_{a b c} \operatorname{Tr}\left(P_{a} P_{b} Q_{c}\right)+\lambda_{2}^{\prime} \operatorname{Tr}\left(Q_{1} R_{2} \Phi_{3}+Q_{2} R_{3} \Phi_{1}\right. \\
& \left.+Q_{3} R_{1} \Phi_{2}\right)+\lambda_{3}^{\prime} \operatorname{Tr}\left(R_{1} R_{2} R_{3}\right) \\
& +\Lambda^{\alpha \beta \gamma} \operatorname{Tr}\left[\left(Q_{1}\right)^{2} S_{\alpha}^{3} T_{\beta}^{1} T_{\gamma}^{2}+\left(Q_{3}\right)^{2} S_{\alpha}^{1} T_{\beta}^{2} T_{\gamma}^{3}\right. \\
& \left.+\left(Q_{2}\right)^{2} S_{\alpha}^{2} T_{\beta}^{3} T_{\gamma}^{1}\right]+\cdots
\end{aligned}
$$

(The notation for the fields are given in Table II.) The couplings $\Lambda^{\alpha \beta \gamma}$ are nonvanishing if the orbifold space group selection rules are satisifed. Here we note that the couplings $\Lambda^{\alpha \beta \gamma}$ for $\alpha, \beta, \gamma$ not all identical are exponentially suppressed in the limit of large volume compactification, whereas the couplings $\Lambda^{\alpha \alpha \alpha}$ are not suppressed. This is because in the former case, the corresponding fields are coming from different fixed points so that upon taking them apart (in the limit of large volume of the orbifold) their coupling becomes weaker and weaker.

Following the discussion in Appendix $\mathrm{C}$ we observe that upon the fields $Q_{a}$ [that are responsible for breaking of the anomalous $\mathrm{U}(1)]$ and $S_{\alpha}^{a}$ (that are the 21 blow-up modes of the $\mathbf{Z}_{7}$ orbifold) acquiring $\mathrm{VEV}$, the states $T_{\alpha}^{a}$ generically become heavy and decouple from the massless spectrum. 
Thus, after blowing up the orbifold singularities on the heterotic side combined with some of the untwisted charged matter fields acquiring VEV's to cancel the $D$-term, we can match the massless spectrum to that of the type I model [where the charged matter must acquire VEV's to cancel the effect of the anomalous U(1)]. Note the crucial role of the perturbative superpotential in this matching. It is precisely such that all the extra fields on the heterotic side can be made massive. Here we note that the blow-up modes $S_{\alpha}^{a}$ are neutral under the anomalous $\mathrm{U}(1)$, and thus do not play an important role in cancelling the $D$-term. (This is to be contrasted with the $Z$-orbifold model discussed in Ref. [1], where the blow-up modes of the $Z$-orbifold carried anomalous $\mathrm{U}(1)$ charge.)

\section{MODULI SPACE}

We now turn to the discussion of the moduli spaces for the type I and heterotic models considered in the previous sections. Let us start with the heterotic model. The (perturbative) moduli space of the corresponding Narain model before orbifolding is $\mathrm{SO}(6,22, \mathbf{Z}) \backslash \mathrm{SO}(6,22) /$ $\mathrm{SO}(6) \otimes \mathrm{SO}(22)$. After orbifolding we have two types of moduli: those coming from the untwisted sector, and those coming from the twisted sector. The untwisted sector moduli parametrize the coset $[\mathrm{SU}(1,3, \mathbf{Z}) \backslash \mathrm{SU}(1,3) / \mathrm{SU}(3) \otimes \mathrm{U}(1)]^{3}$. The subspace $[\mathrm{SU}(1,1, \mathbf{Z}) \backslash \mathrm{SU}(1,1) / \mathrm{U}(1)]^{3}$ of this moduli space is parametrized by six neutral singlets $\phi_{a}$ that correspond to the leftover geometric moduli (coming from the constant metric $g_{i j}$ and antisymmetric tensor $B_{i j}$ fields). The other 12 moduli correspond to the flat directions in the superpotential for the fields $P_{a}, Q_{a}, R_{a}$, and $\Phi_{a}$. (These are the leftover moduli coming from the $6 \times 16$ Wilson lines $A_{i}^{I}$, $I=1, \ldots, 16$, in the original Narain model.)

Next, we turn to the twisted moduli of the heterotic string model. In the twisted sectors, we have the chiral superfields $S_{\alpha}^{a}$ and $T_{\alpha}^{a}$. There is no superpotential for the singlets $S_{\alpha}^{a}$ which are the 21 blow-up modes of the $\mathbf{Z}_{7}$ orbifold. Unlike the $\mathbf{Z}_{3}$ case [1], the blow-up modes are not charged under the anomalous $\mathrm{U}(1)$ and so all of them survive the Higgs process. Notice that both the heterotic and the type I model have anomalous $\mathrm{U}(1)$ with positive trace anomaly. To cancel the $D$-term, one needs to give VEV's to the corresponding negatively charged fields, namely, $Q_{a}$. At a generic point on the heterotic side (i.e., upon giving appropriate VEV's to the untwisted matter fields $Q_{a}$ and the twisted moduli $S_{\alpha}^{a}$ ), the fields $T_{\alpha}^{a}$ become massive (according to the couplings in the superpotential). Thus, the matching is complete after giving appropriate VEV's to both untwisted and twisted fields on the heterotic side, as well as giving appropriate VEV's to open string sector matter fields, and 21 twisted closed string moduli. Upon breaking the anomalous U(1), the dilaton may mix with other gauge singlets. A priori, the mixing is different on the type I and the heterotic side. To make the matching precise, one generically has to appropriately tune the dilaton plus $\phi_{a}$ geometric moduli on both sides.

Let us analyze more carefully how this matching can be achieved. Upon giving VEV to $Q_{1}$, the second and the third $\mathrm{U}(4)$ are broken to the diagonal $\mathrm{U}(4)$. Some of the fields become heavy, whereas $Q_{1}$ is eaten by the super-

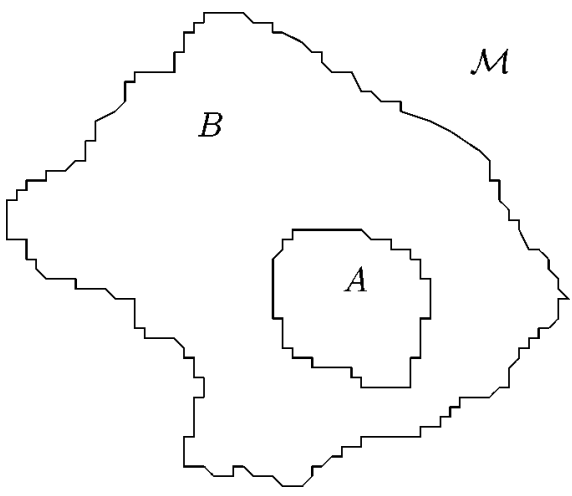

FIG. 1. A schematic picture of the (perturbative) moduli space $\mathcal{M}$ (of the heterotic model). Region A is the subspace corresponding to the type I model. Region B (that complements A in $\mathcal{M}$ ) is the subspace where some or all of the $S_{\alpha}^{a}$ VEV's are zero and some or all of the $T_{\alpha}^{a}$ fields are massless.

Higgs mechanism. The gauge group is further broken to $\mathrm{SU}(4)_{\text {diagonal }} \otimes \mathrm{SO}(8) \otimes \mathrm{U}(1)$ once $Q_{2}$ acquires a nonzero VEV. To break the anomalous U(1), generically, the field $Q_{3}=(\mathbf{6}, \mathbf{1})(-2) \oplus(\overline{\mathbf{1 0}}, \mathbf{1})(-2)$ [in the representations of $\left.\mathrm{SU}(4)_{\text {diagonal }} \otimes \mathrm{SO}(8) \otimes \mathrm{U}(1)\right]$ acquires a VEV. The final gauge group is $\mathrm{Sp}(4)$ or $\mathrm{SO}(4)$ depending on whether $(\mathbf{6}, \mathbf{1})(-2)$ or $(\overline{\mathbf{1 0}}, \mathbf{1})(-2)$ acquires a VEV.

Thus, the moduli spaces (at generic points) of both type I and heterotic models are the same (at the least at tree level). They are described by the untwisted moduli of the heterotic string, or equivalently, the moduli coming from the untwisted closed string sector and the open string sector of the type I model (these parametrize the coset $\left.[\mathrm{SU}(1,3, \mathbf{Z}) \backslash \mathrm{SU}(1,3) / \mathrm{SU}(3) \otimes \mathrm{U}(1)]^{3}\right)$, plus the $2 \times 21$ twisted moduli in the heterotic string model, or equivalently, the moduli coming from the twisted closed string sector of the type I model. The (perturbative) moduli space (of the heterotic model) is schematically depicted in Fig. 1.

It is worth noting the role of anomalous $\mathrm{U}(1)$ in $N=1$ type I-heterotic duality. To cancel the Fayet-Illiopoulos $D$ term, fields that are charged under the anomalous U(1) will generically acquire VEV's. As a result, the extra twisted matter fields in the heterotic model are eliminated via the Higgs mechanism and the matching of the massless spectra of the type I and heterotic models is precise. The appearance of massless twisted matter fields $T_{\alpha}^{a}$ on the heterotic side is a perturbative effect. On the type I side this effect is nonperturbative, and reflects the fact that from the type I point of view there is a (nonperturbative) singularity in the moduli space (or, more precisely, a singular subspace of the full moduli space). Notice that the fields $T_{\alpha}^{a}$ in the heterotic model get heavy via nonrenormalizable terms in the perturbative superpotential. This indicates the importance of perturbative superpotential in $N=1$ type I-heterotic duality.

\section{CONCLUSIONS}

In this paper we discussed a chiral $N=1$ type I model in four dimensions obtained as a compactification on the $\mathbf{Z}_{7}$ orbifold. We studied the type I-heterotic duality in this example, and have concluded that (up to model-dependent differences) the duality in the presence of $D 9$-branes only has a 
clear pattern to it. Note that $\mathbf{Z}_{3}$ (studied in Ref. [1]) and $\mathbf{Z}_{7}$ (studied in this paper) cases exhaust $4 \mathrm{D} \mathbf{Z}_{N}$ orbifolds of type I strings with $N=1$ supersymmetry and no $D 5$-branes. The cases with $D 5$-branes (constructed via orbifolds of even order) are of great interest as they may shed light on nonperturbative dynamics of heterotic five-branes in 4D $N=1$ vacua. Having learned the perturbative part of type I-heterotic duality, now we can march into the more intricate maze of D9- and D5-branes and their (non)perturbative heterotic duals.

\section{ACKNOWLEDGMENTS}

We would like to thank Michael Bershadsky, Peter Cho, Andrei Johansen, Joe Polchinski, John Schwarz, Matthew Strassler, Tom Taylor, Henry Tye, and Cumrun Vafa for discussions. The research of G.S. was partially supported by the National Science Foundation. G.S. would also like to thank Joyce M. Kuok Foundation for financial support. The work of Z.K. was supported in part by Grant No. NSF PHY96-02074, and the U.S. DOE. Z.K. would also like to thank Albert and Ribena $\mathrm{Yu}$ for financial support.

\section{APPENDIX A: TADPOLES FOR ORBIFOLD SINGULARITIES}

In this appendix we discuss the tadpole cancellation constraints for orbifold compactifications of type I strings. We confine our attention to $\mathbf{Z}_{N}$ orbifolds without D5-branes. This means that $N$ is odd, and without loss of generality we can take $N$ to be a prime number (as all the $\mathbf{Z}_{N}$ orbifold cases with $N=1$ supersymmetry and no $D 5$-branes in six and four dimensions are restricted to prime $N$ ). The constraints that we present here can be easily generalized to other cases (including those with $D 5$-branes), which will be discussed elsewhere.

There are two kinds of constraints we need to consider. The first one comes from the cancellation of the untwisted tadpoles for the $D 9$-branes. This constraint is the same in all dimensions and leads to the statement that there are 16 D9-branes not counting the orientifold images. (This last statement is only correct if the NS-NS antisymmetric background $B_{i j}$ is set equal to zero; see below.) The other constraint comes from the cancellation of the twisted tadpoles for the D9-branes. The twisted tadpoles have been computed in six dimensions in Ref. [10] (for the $\mathbf{Z}_{2}$ orbifold limit of $K 3$ ) and Ref. [11] (for all the other orbifold limits of $K 3$ ), and in four dimensions for the $\mathbf{Z}_{2} \otimes \mathbf{Z}_{2}$ orbifold [4] and the $\mathbf{Z}_{3}$ orbifold [2]. For the case we are considering here (odd prime $N$ ), there is a simple formula which expresses the twisted tadpole cancellation condition that we are now going to discuss.

Let us be general here and consider compactification on $T^{2 d} / \mathbf{Z}_{N}$ with the twist given by

$$
T_{N}=\left(t_{1}, t_{2}, \ldots, t_{d} \| t_{1}, t_{2}, \ldots, t_{d}\right)
$$

Here $t_{i}$ are fractional numbers taking values in $\{0,1 / N, 2 / N, \ldots,(N-1) / N\}$. A given $t_{i}$ corresponds to a twist of the $i$ th complex boson by a $2 \pi t_{i}$ rotation. (We have complexified the $2 d$ real bosons into $d$ complex bosons).
The double vertical line separates the right and left movers of the string. Because we are considering symmetric orbifold, the right- and left-moving twists are the same. Also note that the consistency of the orbifold requires that the expression

$$
\prod_{i=1}^{d} 4 \sin ^{2}\left(\pi t_{i}\right)
$$

where the factors with $t_{i}=0$ are not included in the product, be an integer. In fact the latter is nothing but the number of fixed points (tori) in the $T_{N}$ twisted sector.

The orbifold action on Chan-Paton factors is described by the unitary matrices $\gamma$ that act on the string end points. In our case $\gamma$ (we are suppressing all the indices as they are straightforward to reconstruct) is a $16 \times 16$ matrix (note that it is not a $32 \times 32$ matrix because we have chosen not to count the orientifold images of the $D 9$-branes). We can diagonalize this matrix. Then, the most general form of this matrix is given by

$$
\gamma=\bigotimes_{k=0}^{N-1} \omega^{k} \mathbf{I}_{m_{k}} .
$$

Here $\omega \equiv \exp (2 \pi i / N)$ and $\mathbf{I}_{m_{k}}$ is the $m_{k} \times m_{k}$ unit matrix. Note that $\sum_{k=0}^{N-1} m_{k}=16$.

The twisted tadpole cancellation condition in our notation reads

$$
\operatorname{Tr}(\gamma)=16 p, \quad p \equiv \prod_{i=1}^{d}(-1)^{N t_{i}} \cos \left(\pi t_{i}\right)
$$

Note that for this equation to have a solution, we must put $m_{1}=m_{2}=\cdots=m_{N-1} \equiv m$. [Also note that $m_{0}=16$ $-m(N-1) \equiv n$.] Then $\operatorname{Tr}(\gamma)=16-N m$ (note that $\left.\sum_{k=1}^{N-1} \omega^{k}=-1\right)$.

The gauge group of the model can be easily seen from the above tadpole equation. It is given by $\mathrm{U}(2 m)^{(N-1) / 2}$ $\otimes \operatorname{SO}(2 n)$, where

$$
m=16(1-p) / N, \quad n=16[1+(N-1) p] / N .
$$

Note that if none of the $t_{i}$ are zero, then $|p|=2^{-d}$.

Let us illustrate these equations with a few examples.

$6 \mathrm{D} \mathbf{Z}_{3}$ orbifold (i.e., $\mathbf{Z}_{3}$ orbifold limit of $K 3$ ). The twist reads

$$
T_{3}=(1 / 3,1 / 3|| 1 / 3,1 / 3)
$$

Note that $p=+1 / 4$ in this case, $m=4$ and $n=8$, so that the gauge group is $\mathrm{U}(8) \otimes \mathrm{SO}(16)$. This is the model considered in Ref. [11].

4D $\mathbf{Z}_{3}$ orbifold (i.e., Z-orbifold limit of a Calabi-Yau threefold). The twist read:

$$
T_{3}=(1 / 3,1 / 3,1 / 3|| 1 / 3,1 / 3,1 / 3) .
$$

Note that $p=-1 / 8$ in this case, $m=6$ and $n=4$, so that the gauge group is $\mathrm{U}(12) \otimes \mathrm{SO}(8)$. This is the model considered in Ref. [2]. 
4D $\mathbf{Z}_{7}$ orbifold (i.e., $\mathbf{Z}_{7}$ orbifold limit of a Calabi-Yau threefold). The twist reads

$$
T_{7}=(1 / 7,2 / 7,3 / 7 \| 1 / 7,2 / 7,3 / 7) .
$$

Note that $p=+1 / 8$ in this case, $m=2$ and $n=4$, so that the gauge group is $\mathrm{U}(4) \otimes \mathrm{U}(4) \otimes \mathrm{U}(4) \otimes \mathrm{SO}(8)$. This is the model considered in this paper.

Here we also give two nonsupersymmetric models (that have never been discussed previously to the best of our knowledge).

$6 \mathrm{D} \mathbf{Z}_{5}$ orbifold (i.e., compactification on $T^{4} / \mathbf{Z}_{5}$ ). The twist reads

$$
T_{5}=(1 / 5,2 / 5|| 1 / 5,2 / 5)
$$

Note that $p=-1 / 4$ in this case, $m=4$ and $n=0$, so that the gauge group is $\mathrm{U}(8) \otimes \mathrm{U}(8)$.

8D $\mathbf{Z}_{3}$ orbifold (i.e., compactification on $T^{2} / \mathbf{Z}_{3}$ ). The twist reads

$$
T_{3}=(1 / 3 \| 1 / 3)
$$

Note that $p=-1 / 2$ in this case, $m=8$ and $n=0$, so that the gauge group is $\mathrm{U}(16)$.

Finally, we would like to consider the cases with nonzero NS-NS antisymmetric background $B_{i j}$. Although there are no massless scalars corresponding to these in type I theory (recall that there $B_{i j}$ fields are projected out of the spectrum after orientifolding), i.e., these moduli cannot be varied continuously, they can have certain quantized values (because of this they are not moduli in the conventional sense of this word). The quantization is due to the fact that to have a consistent orientifold the corresponding type IIB spectrum must be left-right symmetric. At generic values of $B_{i j}$ this symmetry is destroyed. There are, however, certain discrete $B_{i j}$ backgrounds compatible with the orientifold projection [12]. The effect of nonzero $B_{i j}$ background is that the rank of the gauge group coming from the $\mathrm{SO}(32)$ (i.e., Chan-Paton) factor is reduced, depending on the rank $r$ (which is always even) of the matrix $B_{i j}$. That is, the number of the $D 9$-branes required by the tadpole cancellation condition is no longer 16 but $16 / 2^{r / 2}$. All of the above formulas then get modified in the presence of rank $r B_{i j}$ in an obvious way via replacing the factor 16 everywhere by $16 / 2^{r / 2}$.

As mentioned earlier, it is not difficult to generalize the tadpole cancellation condition discussed in this appendix to the cases with D5-branes. The work on these cases is in progress and will be reported elsewhere. (It would also be interesting to generalize the above conditions along the lines of Ref. [13].)

\section{APPENDIX B: BOSONIC SUPERCURRENT AND SCATTERING}

In this appendix, we review the bosonic supercurrent approach in calculating scattering amplitudes of orbifold models [5]. The basic idea of this approach is that at the enhanced symmetry point, we can rewrite the twists as shifts in the momentum lattice. The twist fields can be expressed in terms of ordinary momentum states; their quantum numbers are straightforward to identify and their correlation functions are easy to calculate.

To be specific, let us focus on four-dimensional heterotic string models within the framework of conformal field theory and consider only Abelian orbifolds. Before orbifolding, the corresponding Narain model has $N=4$ space-time supersymmetry and the internal momenta span an even selfdual Lorentzian lattice $\Gamma^{6,22}=\Gamma^{6,6} \oplus \Gamma^{16}$. Let $X(\bar{z})$ be one of the three right-moving complex bosons corresponding to the six compactified dimensions in $\Gamma^{6,22}$. In terms of two real bosons, $X=\left(X_{1}+i X_{2}\right) / \sqrt{2}$. For a $\mathbf{Z}_{N}$ twist (for simplicity, $N$ is taken to be prime), in the neighborhood of a twist field located at the origin, $X(\bar{z})$ undergoes a phase rotation:

$$
\partial X\left(\bar{z} e^{-2 \pi i}\right)=\exp (-2 \pi i k / N) \partial X(\bar{z})
$$

which is called the monodromy of $X(\bar{z})$. (Note that $k$ is an integer.) The basic twist field $\sigma(\bar{z})$ has conformal weight $h=k(1-k / N) / 2 N$. It twists $X(\bar{z})$ by $\exp (-2 \pi i k / N)$ and its complex conjugate $\bar{X}(\bar{z})$ by $\exp (2 \pi i k / N)$; i.e., their operator product expansions (OPE's) are [9]

$$
\begin{gathered}
i \partial X(\bar{z}) \sigma(\bar{w})=(\bar{z}-\bar{w})^{-(1-k / N)} \tau(\bar{w})+\cdots, \\
i \partial \bar{X}(\bar{z}) \sigma(\bar{w})=(\bar{z}-\bar{w})^{-k / N} \tau^{\prime}(\bar{w})+\cdots,
\end{gathered}
$$

where $\tau$ and $\tau^{\prime}$ are excited twist fields.

In this paper, we consider compactification on a $\mathbf{Z}_{7}$ orbifold. The lattice $\Gamma^{6,6}$ must have a $\mathbf{Z}_{7}$ symmetry. At generic points with this symmetry the gauge symmetry (of the Narain model) coming from $\Gamma^{6,6}$ is $\mathrm{U}(1)^{6}$. This symmetry is enhanced to $\mathrm{SU}(7)$ at the special point. In terms of six real bosons $\phi^{I}$, the Cartan generators are $i \partial \phi^{I}$, whereas the root generators are $J_{Q}=\exp (i Q \cdot \phi) c(Q)$. Here, we have introduced six-dimensional real vectors $Q=\left(Q^{1}, \ldots, Q^{6}\right)$ which are root vectors of $\mathrm{SU}(7)$ with length squared 2 . The $c(Q)$ are cocycle operators necessary in the Kac-Moody algebra. For convenience, we shall not always explicitly display $c(Q)$ : their presence is understood.

Suppose we can rewrite each $i \partial X^{a}$ (where $X^{a}$ are the three right-moving complex bosons corresponding to $\Gamma^{6,6}$ ) as a sum of the root generators:

$$
i \partial X^{a}=\sum_{Q^{2}=2} \xi^{a}(Q) J_{Q}, \quad a=1,2,3 .
$$

Then a twist on $\partial X^{a}$ in Eq. (B1) becomes a shift in $\phi^{I}$ :

$$
\phi^{I}\left(\bar{z} e^{-2 \pi i}\right)=\phi^{I}(\bar{z})-2 \pi U^{I}
$$

where $Q \cdot U=k / N$. The coefficients $\xi^{a}(Q)$ must be chosen such that the following OPE's are satisfied:

$$
\begin{gathered}
\partial X^{a}(\bar{z}) \partial X^{b}(0) \sim \text { regular, } \\
\partial X^{a}(\bar{z}) \partial X^{b \dagger}(0) \sim-\bar{z}^{-2} \delta^{a b}+\text { regular. }
\end{gathered}
$$

For a lattice with $\mathrm{SU}(N)$ enhanced symmetry $(N=7$ in our case), the choice is unique (up to equivalent representations): 


$$
i \partial X=\frac{1}{\sqrt{N}} \sum_{\alpha=1}^{N} e^{-i e_{\alpha} \cdot \phi}, \quad i \partial \bar{X}=\frac{1}{\sqrt{N}} \sum_{\alpha=1}^{N} e^{i e_{\alpha} \cdot \phi}
$$

where $e_{\alpha}$ for $\alpha=1, \ldots, N-1$ are simple roots of $\mathrm{SU}(N)$ and $e_{N}=-\sum_{\alpha=1}^{N-1} e_{\alpha}$. We have suppressed the index $a$. The set of roots chosen depends on the monodromy of $i \partial X^{a}$ and can be different for $a=1,2,3$.

To see explicitly how this conversion of twists to shifts can be realized, let us consider the decomposition of $\mathbf{N}$ of SU $(N)$ into representations of $\mathrm{U}(1)^{N-1}$ :

$$
\begin{aligned}
\mathbf{N}= & (1, \ldots, 1) \oplus(-1,1, \ldots, 1) \oplus(0,-2,1, \ldots, 1) \\
& \oplus(0,0,-3,1, \ldots, 1) \oplus \ldots \oplus(0,0, \ldots,-N+1) .
\end{aligned}
$$

The normalization radii of $\mathrm{U}(1)^{N-1}$ are given by $(1 / \sqrt{(1) 2}, 1 / \sqrt{(2) 3}, \ldots, 1 / \sqrt{(N-1) N)}$. They are chosen such that all the states on the right-handed side of the equation have the same conformal dimension as $\mathbf{N}$ of $\mathrm{SU}(N)$, i.e., $h=(N-1) / 2 N$. The conjugate representation $\overline{\mathbf{N}}$ has opposite $\mathrm{U}(1)^{N-1}$ charges. The adjoint (and hence the roots $Q$ ) of $\mathrm{SU}(N)$ can be obtained from the tensor product $\mathbf{N} \otimes \overline{\mathbf{N}}$.

To construct the shift representation $U$ of the twisted fields in the singly twisted sector, we demand that the $N$ states in Eq. (B7) pick up different phases under the shift (i.e., $Q \cdot U=k / N$ for $k=0,1, \ldots, N-1$ ). The shift representation is uniquely determined once we fix the assignment of the phases. The roots of $\mathrm{SU}(N)$ can be divided into sets with different phases under the shift $U$. Terms that appear in Eq. (B6) are determined by the monodromy of $i \partial X$. [Thus, for $N=7$ we have 42 roots. $21=3 \times 7$ enter in the expressions (B6) for the three bosons $i \partial X^{a}$, and the other $21=3 \times 7$ roots enter in the expressions (B6) for the three bosons (conjugate) $i \partial \bar{X}^{a}$.]

For prime $N$, there are $N$ twisted sectors, each with $N$ fixed points. To obtain the singly twisted fields corresponding to the other fixed points, we simply add weights of $\mathrm{SU}(N)$ to $U$ such that the conformal dimension is preserved, i.e., $1 / 2(U+W)^{2}=1 / 2 U^{2}$. There is precisely one weight vector in each unitary representation of $\mathrm{SU}(N)$ that satisfies the above requirement, and there are $N$ unitary representations of $\mathrm{SU}(N)$ : the identity $\mathbf{1}$ and the antisymmetric tensors $\epsilon^{i j \cdots k} \mathbf{N}_{i} \otimes \mathbf{N}_{j} \otimes \cdots \otimes \mathbf{N}_{k}$. [In the case of SU(7), they are the identity $\mathbf{1}$, the weights $\mathbf{7}, \mathbf{2 1}, \mathbf{3 5}$, and their conjugate weight representations.] The twisted fields in higher twisted sectors are represented by the shifts $k U+Q$ where $k=2, \ldots, N-1$ and $Q$ is a root vector added so as to preserve the conformal dimension of the twisted states. Again, the higher twisted fields at other fixed points are obtained by adding appropriate weights.

In the standard orbifold formalism, the internal part of the supercurrent for the right movers can be written as

$$
T_{F}=\frac{i}{2} \sum_{a=1}^{3} \psi^{a} \partial X^{a}+\text { H.c. }
$$

where $\psi^{a}$ are complex world-sheet fermions. The twists on $\psi^{a}$ can be written as shifts if we bosonize the complex fermions:

$$
\begin{gathered}
\psi^{a}=\exp \left(i \rho^{a}\right)=\exp (i H \cdot \rho), \\
\psi^{a \dagger}=\exp \left(-i \rho^{a}\right)=\exp (-i H \cdot \rho) .
\end{gathered}
$$

where $H$ (known as the $H$ charge) equals $(1,0,0),(0,1,0)$, or $(0,0,1)$ for $a=1,2,3$.

The bosonic supercurrent is then given by (the cocycle operators are not displayed)

$$
T_{F}=\frac{1}{2 \sqrt{N}} \sum_{a=1}^{3} e^{i \rho^{a}} \sum_{\alpha=1}^{N} e^{i Q_{\alpha}^{a} \cdot \phi}+\text { H.c. . }
$$

The supercurrent is therefore a linear combination of terms with well defined $H$ and $Q$-charges.

In the covariant gauge, we have the reparametrization ghosts $b$ and $c$, and superconformal ghosts $\beta$ and $\gamma$ [14]. It is most convenient to bosonize the $\beta, \gamma$ ghosts:

$$
\beta=\partial \xi e^{-\phi}, \quad \gamma=\eta e^{\phi},
$$

where $\xi$ and $\eta$ are auxiliary fermions and $\phi$ is a bosonic ghost field obeying the OPE $\phi(\bar{z}) \phi(\bar{w}) \sim \ln (\bar{z}-\bar{w})$. The conformal dimension of $e^{q \phi}$ is $-1 / 2 q(q+2)$. In covariant gauge, vertex operators are of the form $V(z, \bar{z})=V(z) \bar{V}(\bar{z})$, where $V(z)$ and $V(\bar{z})$ are both dimension 1 operators constructed from the conformal fields. These include the longitudinal components as well as the ghosts. The vertex operators for space-time bosons carry integral ghost charges ( $q$ $\in \mathbf{Z}$ ) whereas for space-time fermions the ghost charges are half-integral $(q \in \mathbf{Z}+1 / 2)$. Here, $q$ specifies the picture. The canonical choice is $q=-1$ for space-time bosons and $q=-1 / 2$ for space-time fermions. We will denote the corresponding vertex operators by $V_{-1}(z, \bar{z})$ and $V_{-1 / 2}(z, \bar{z})$, respectively. Vertex operators in the $q=0$ picture (with zero ghost charge) is given by picture changing :

$$
V_{0}(z, \bar{z})=\lim _{\bar{w} \rightarrow \bar{z}} e^{\phi} T_{F}(\bar{z}) V_{-1}(z, \bar{w})
$$

Having constructed the vertex operators for the massless states, one can in principle compute the scattering amplitudes, or the corresponding couplings in the superpotential. The coupling of $M$ chiral superfields in the superpotential is given by the scattering amplitude of the component fields in the limit when all the external momenta are zero. Due to holomorphicity, one needs to consider only the scatterings of left-handed space-time fermions, with vertices $V_{-1 / 2}(z, \bar{z})$, and their space-time superpartners. Since the total $\phi$ ghost charge in any tree-level correlation function is -2 , it is convenient to choose two of the vertex operators in the $-1 / 2$-picture, one in the -1 picture, and the rest in the 0 picture. Using the SL(2,C) invariance, the scattering amplitude is therefore

$$
\begin{aligned}
\mathcal{A}_{M}= & g_{\mathrm{st}}^{M-2} \int d z_{4} d \bar{z}_{4} \cdots d z_{M} d \bar{z}_{M} \\
& \times\left\langle V_{-1 / 2}(0,0) V_{-1 / 2}(1,1) V_{-1}(\infty, \infty)\right. \\
& \left.\times V_{0}\left(z_{4}, \bar{z}_{4}\right) \cdots V_{0}\left(z_{M}, \bar{z}_{M}\right)\right\rangle,
\end{aligned}
$$


TABLE III. The $\mathbf{Q}_{R}$ and $\mathbf{Q}_{L}$ charges for the untwisted sector fields $\phi_{\alpha}^{a}$. The $\mathrm{U}(1)_{R}^{6}$ and $\mathrm{U}(1)_{L}^{6}$ normalization radii are given at the bottom of the table.

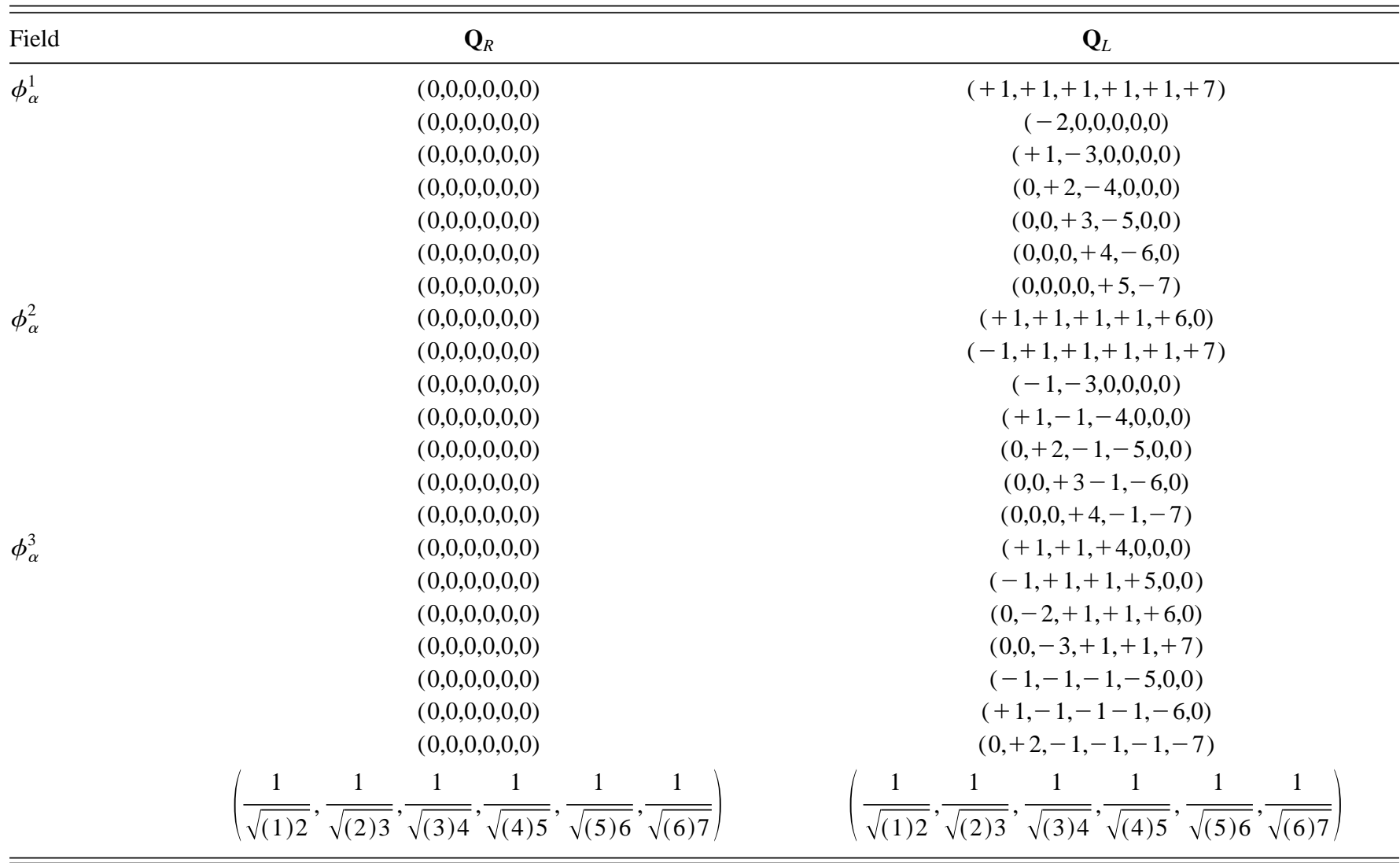

where we have normalized the $c$ ghost part of the correlation function $\langle c(0,0) c(1,1) c(\infty, \infty)\rangle$ to 1 . For a nonzero coupling, the sum of the $H$ charges as well as the sum of the $Q$ charges must be zero in the corresponding scattering amplitude. Note that the supercurrent carries terms with different $H$ and $Q$ charges. Because of picture changing, $H$ and $Q$ charges are not global charges even though they must be conserved exactly. Point group and space group selection rules follow from these conservation laws.

\section{APPENDIX C: $Z_{7}$ ORBIFOLD SPACE GROUP SELECTION RULES}

In this appendix we derive the space group selection rules for the symmetric $\mathbf{Z}_{7}$ orbifold. A priori, one needs to understand the scattering of the primary twist fields and their descendents to solve this problem. The general framework for calculating scatterings of twisted fields in orbifolds have been developed in Ref. [9] using techniques in conformal field theory. The actual calculations, however, can be quite nontrivial. Fortunately, there exists a simpler way of deducing the space group selection rules. This other way utilizes the techniques recently developed in Ref. [5] based on the bosonic supercurrent framework discussed in Appendix B. The idea is to compute the scattering at the enhanced gauge symmetry point where the vertex operators for all the twist fields (up to cocycles) can be expressed as exponentials of the $\mathbf{Q}_{R}$ and $\mathbf{Q}_{L}$ charges (see Appendix B), hence the problem can be solved relatively easily. At the enhanced gauge symmetry point in the moduli space the orbifold selection rules are given by the conservation of gauge charges $\mathbf{Q}_{L}$, and also by the conservation of the $\mathbf{Q}_{R}$ and $H$ charges. (Both of these are affected by picture changing, and therefore are neither local nor global charges in space-time.) Ultimately, we would like to obtain the orbifold space group selection rules away from the enhanced symmetry point. This can be done by considering the corresponding couplings within effective field theory, and subsequently breaking the enhanced gauge symmetry by giving VEV's to the corresponding scalars. The latter procedure is an effective field theory manifestation of tuning the stringy moduli away from the enhanced symmetry point. Once the enhanced gauge symmetry is broken completely, we obtain the space group selection rules (i.e., the knowledge of whether a given coupling vanishes or not according to this discrete symmetry) by simply examining the superpotential. Note that at the enhanced symmetry point there are a number of fields in the untwisted sector charged under the enhanced gauge symmetry but neutral under the original one. Upon using the Higgs mechanism on the enhanced gauge symmetry completely, some of them are eaten in the super-Higgs mechanism, and some of them acquire masses via the tree-level superpotential. As a result, the number of neutral scalars is precisely equal to the dimension of the space parametrized by the geometric moduli of the orbifold. This is to be contrasted with the fact that in the twisted sectors the number of fields does not depend upon the values of the geometric moduli whether they are at a generic or enhanced symmetry point.

Thus, let us start from the Narain model with $N=4$ space- 
TABLE IV. The $\mathbf{Q}_{R}$ and $\mathbf{Q}_{L}$ charges for the twisted sector fields $T_{\alpha}^{a}$. The $\mathrm{U}(1)_{R}^{6}$ and $\mathrm{U}(1)_{L}^{6}$ normalization radii are given at the bottom of the table.

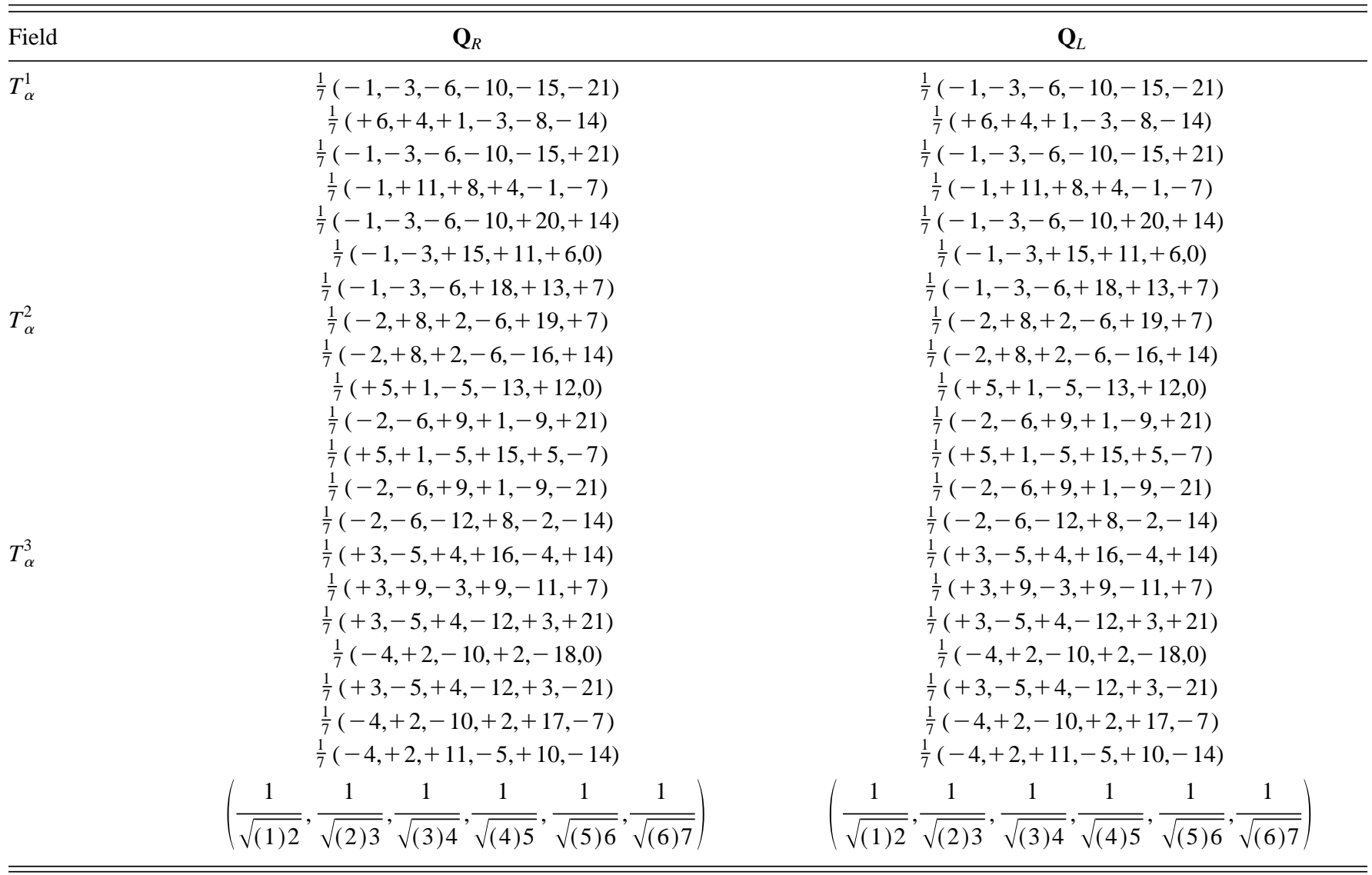

time supersymmetry in four dimensions. Let the momenta of the internal (6 right-moving and 22 left-moving) world-sheet bosons span the (even self-dual) Narain lattice $\Gamma^{6,22}=\Gamma^{6,6} \otimes \Gamma^{16}$. Here $\Gamma^{16}$ is the $\operatorname{spin}(32) / \mathbf{Z}_{2}$ lattice, whereas the lattice $\Gamma^{6,6}$ is spanned by the momenta $\left(p_{R}|| p_{L}\right)$ with $\left.p_{L}, p_{R} \in \Gamma\right][\mathrm{SU}(7)$ weight lattice $]$, and $p_{L}-p_{R} \in \Gamma^{7}$ [SU(7) root lattice]. Note that this corresponds to a compactification on a six-torus with spacial values of the constant background metric $g_{i j}$ and (nonzero) antisymmetric tensor $B_{i j}$. This Narain model has gauge group $\mathrm{SU}(7) \otimes \mathrm{SO}(32)$. The first factor SU(7) comes from the oscillator excitations and momentum states of the left-moving world-sheet bosons corresponding to $\Gamma^{6,6}$ (i.e, the six-torus). The second factor $\mathrm{SO}(32)$ comes from the other 16 left-moving world-sheet bosons.

Next consider the $\mathbf{Z}_{7}$ orbifold model (with nonstandard embedding of the gauge connection) obtained via twisting the above Narain model by the twist $T_{7}$ given in Sec. III. This model has $N=1$ supersymmetry, and gauge group $\mathrm{U}(1)^{6} \otimes\left[\mathrm{U}(4)^{3} \otimes \mathrm{SO}(8)\right]$. The factor $\mathrm{U}(4)^{3} \otimes \mathrm{SO}(8)$ comes from the breaking of $\mathrm{SO}(32)$. The factor $\mathrm{U}(1)^{6}$ comes from the breaking of SU(7). Note that, as discussed in Appendix $\mathrm{B}$, we can represent the $\mathbf{Z}_{7}$ twist $\left(\theta, \theta^{2}, \theta^{3} \| \theta, \theta^{2}, \theta^{3}\right)$ acting in the six-torus in terms of a $\mathbf{Z}_{7}$ (i.e., order 7 ) shift provided that the right-moving supercurrent is written in the bosonized form. Here we give this shift in terms of the $\mathrm{SU}(7) \supset \mathrm{U}(1)^{6}$ basis, where the normalization radii of the six U(1)'s are given by $(1 / \sqrt{(1) 2}, 1 / \sqrt{(2) 3}, 1 / \sqrt{(3) 4}, 1 / \sqrt{(4) 5}, 1 / \sqrt{(5) 6}$,
$1 / \sqrt{(6) 7})$. Thus, in this basis the twist $T_{7}$ is replaced by the shift

$$
\begin{aligned}
T_{7}^{\prime}= & {\left[-\frac{1}{7},-\frac{3}{7}-\frac{6}{7},-\frac{10}{7},-\frac{15}{7},-\frac{21}{7} \|-\frac{1}{7},-\frac{3}{7}-\frac{6}{7},\right.} \\
& \left.-\frac{10}{7},-\frac{15}{7},-\frac{21}{7} \mid\left(\frac{1}{7}\right)^{4}\left(\frac{2}{7}\right)^{4}\left(\frac{3}{7}\right)^{4} 0^{4}\right] .
\end{aligned}
$$

In this basis it is straightforward to work out the $\mathbf{Q}_{R}$ and $\mathbf{Q}_{L}$ charges of the massless states of the model. The latter are the same as in the model discussed in Sec. III, except for the untwisted sector singlets. Thus, instead of three neutral singlets $\phi_{a}$ (see Table II) we have 21 fields $\phi_{\alpha}^{a}, \alpha=1, \ldots, 7$, that are singlets under $\mathrm{U}(4)^{3} \otimes \mathrm{SO}(8)$ gauge group, but are charged under $\mathrm{U}(1)^{6}$ Abelian subgroup. Their charges are given in Table III. By giving VEV's to these singlets we can completely break U(1) ${ }^{6}$ gauge symmetry. Due to the superHiggs mechanism and the corresponding superpotential after using the Higgs mechanism, only three neutral fields $\phi_{a}$ survive in the massless spectrum. The rest are either eaten by the gauge bosons or become heavy via the couplings in the superpotential. This field theory breaking is in one-to-one correspondence with the string theory picture of moving in the moduli space $[\mathrm{SU}(1,1, \mathbf{Z}) \backslash \mathrm{SU}(1,1) / \mathrm{U}(1)]^{3}$ discussed earlier. That is, we are moving the moduli away from the special point of enhanced gauge symmetry into the bulk, i.e., to some generic point.

The bosonic supercurrent is given by 
TABLE V. The $\mathbf{Q}_{R}$ and $\mathbf{Q}_{L}$ charges for the twisted sector fields and $S_{\alpha}^{a}$. The $\mathrm{U}(1)_{R}^{6}$ and $\mathrm{U}(1)_{L}^{6}$ normalization radii are given at the bottom of the table.

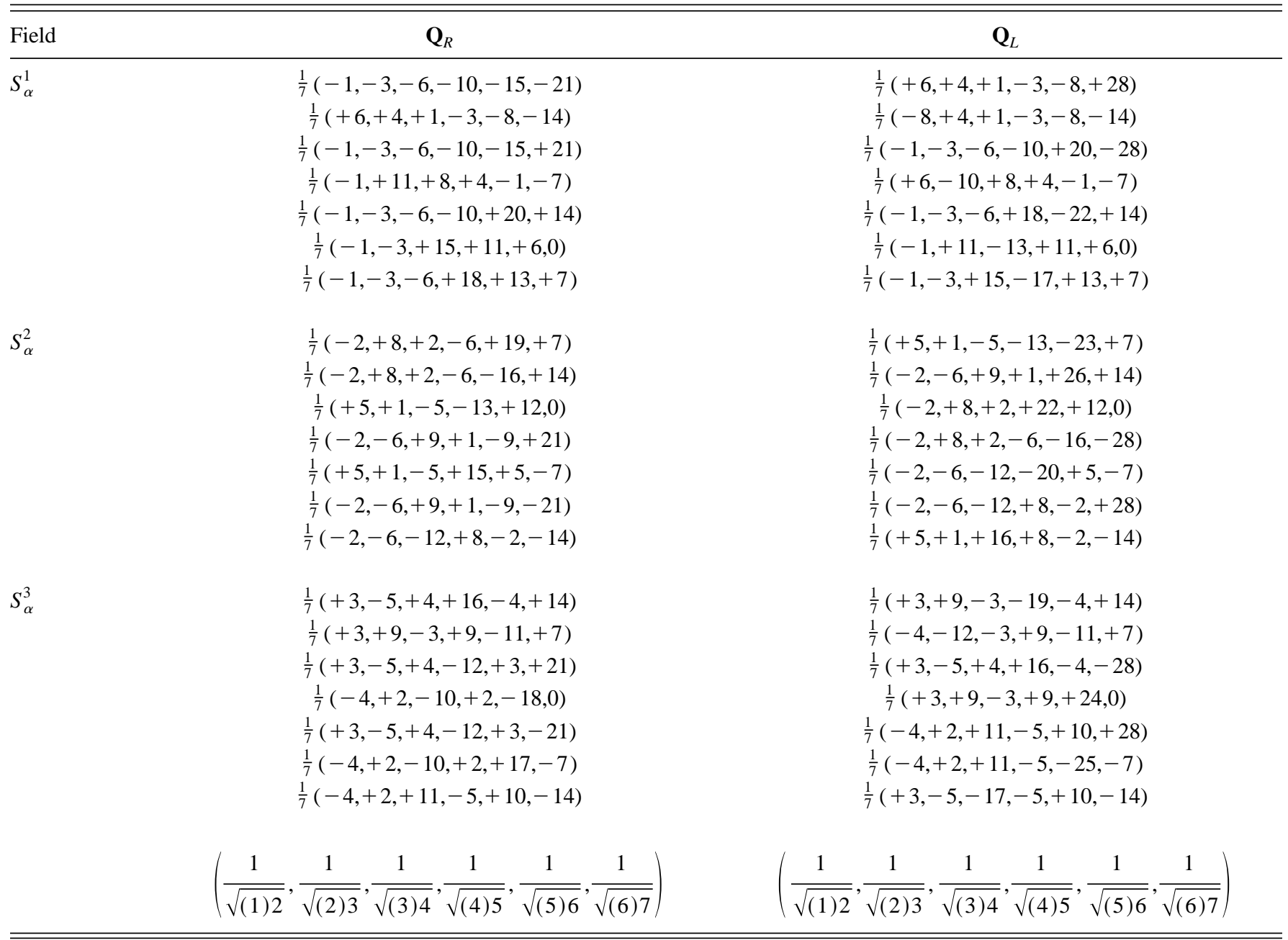

$$
\begin{aligned}
T_{F}= & \frac{1}{2 \sqrt{7}}\left(e^{i \rho_{1}} \sum_{\alpha=1}^{7} e^{i Q_{\alpha}^{1} \cdot \phi}+e^{i \rho_{2}} \sum_{\alpha=1}^{7} e^{i Q_{\alpha}^{2} \cdot \phi}\right. \\
& \left.+e^{i \rho_{3}} \sum_{\alpha=1}^{7} e^{-i Q_{\alpha}^{3} \cdot \phi}\right)+ \text { H.c. }
\end{aligned}
$$

where the $Q_{\alpha}^{a}$ charges for the currents $i \partial X^{a}$ are the same as the $\mathbf{Q}_{L}$ charges for the fields $\phi_{\alpha}^{a}$ in Table III (and this is no coincidence for the orbifold is symmetric).

Note that the untwisted sector fields $P_{a}, Q_{a}, R_{a}$ and $\Phi_{a}$ are not charged under the enhanced $\mathrm{U}(1)^{6}$ gauge symmetry, so that the couplings $\lambda_{1,2,3}$ for the untwisted sector fields do not vanish at any point in the moduli space (but smoothly vary with the moduli). The twisted sector fields $T_{\alpha}^{a}$ and $S_{\alpha}^{a}$ do carry $\mathrm{U}(1)^{6}$ charges. The $\mathrm{U}(1)^{6}$ charges along with the $\mathbf{Q}_{R}$ charges for the fields $T_{\alpha}^{a}$ and $S_{\alpha}^{a}$ are given in Tables IV and Table V. Because $T_{\alpha}^{a}$ and $S_{\alpha}^{a}$ carry $\mathrm{U}(1)^{6}$ charges, some of the couplings $\Lambda^{\alpha \beta \gamma}$ that are nonzero at generic points vanish at the enhanced gauge symmetry point.

Since the model possesses explicit $\mathbf{Z}_{3}$ cyclic symmetry $(a=1) \rightarrow(a=2) \rightarrow(a=3) \rightarrow(a=1)$, we can confine our attention to couplings $\left(Q_{1}\right)^{2} T_{\alpha}^{1} T_{\beta}^{2} S_{\gamma}^{3}$. For example, according to Tables III, IV and $\mathrm{V}$, the coupling $\left(Q_{1}\right)^{2} T_{\alpha}^{1} T_{\beta}^{2} S_{\gamma}^{3}$ for $\alpha=1, \beta=\gamma=4$ is allowed by $\mathbf{Q}_{R}$ (here one needs to take into account the picture changing) and $\mathbf{Q}_{L}$ charge conservation. On the other hand, say, the coupling with $\alpha=\beta=\gamma=1$ is not allowed. There is, however, a higher point coupling, namely, $\left(Q_{1}\right)^{2} T_{1}^{1} T_{1}^{2} S_{1}^{3} \phi_{3}^{1} \phi_{2}^{3}$ that is allowed. Upon the fields $\phi_{3}^{1}$ and $\phi_{2}^{3}$ acquiring VEV's, we, therefore, have an effective coupling $\left(Q_{1}\right)^{2} T_{1}^{1} T_{1}^{2} S_{1}^{3}$. From examining the $\mathbf{Q}_{R}$ and $\mathbf{Q}_{L}$ charge conservation in the scattering of states $\left(Q_{1}\right)^{2} T_{\alpha}^{1} T_{\beta}^{2} S_{\gamma}^{3}$, it becomes clear that near the enhanced symmetry point upon the fields $S_{\alpha}^{3}$ and $Q_{1}$ acquiring VEV's, all the fields $T_{\alpha}^{1}$ and $T_{\beta}^{2}$ generically become massive. Similarly, if all the VEV's $Q_{a}$ and $S_{\alpha}^{a}$ are nonzero, all the fields $T_{\alpha}^{a}$ are generically massive. In fact, this conclusion does not depend on being close to the enhanced symmetry point. Thus, consider the basis for $\alpha, \beta, \gamma$ indices such that they label the fixed points of the orbifold [this basis is not the same as that of $\mathrm{SU}(7) \supset \mathrm{U}(1)^{6}$, but can be constructed from the latter via a rotation]. Then it is clear that in the limit of large volume of the orbifold the couplings $\Lambda^{\alpha \beta \gamma}$ for $\alpha, \beta, \gamma$ are exponentially suppressed, whereas the couplings $\Lambda^{\alpha \alpha \alpha}$ are not. The latter couplings are nonzero at generic points in the moduli space. From this it should become clear that generically all the fields $T_{\alpha}^{a}$ are heavy as long as we turn on vacuum expectation values (VEV's) for all of the fields $Q_{a}$, and also $S_{\alpha}^{a}$. 
[1] Z. Kakushadze, " Aspects of $N=1$ Type I-Heterotic Duality in Four Dimensions,” hep-th/9704059.

[2] C. Angelantonj, M. Bianchi, G. Pradisi, A. Sagnotti, and Ya. S. Stanev, Phys. Lett. B 385, 96 (1996).

[3] J. Polchinski and E. Witten, Nucl. Phys. B460, 525 (1996).

[4] M. Berkooz and R. Leigh, Nucl. Phys. B483, 187 (1997).

[5] Z. Kakushadze, G. Shiu, and S.-H.H. Tye, Nucl. Phys. B (to be published).

[6] S. Sethi, C. Vafa, and E. Witten, Nucl. Phys. B480, 213 (1996). R. Friedman, J. Morgan, and E. Witten, Commun. Math. Phys. 187, 679 (1997); M. Bershadsky, A. Johansen, T. Pantev, and V. Sadov, "Four-Dimensional Compactifications of $F$-theory,' hep-th/9701165.

[7] M. Green and J. Schwarz, Phys. Lett. 149B, 117 (1984); 151B, $21(1985)$
[8] E. Witten, Phys. Lett. 149B, 351 (1984); M. Dine, N. Seiberg, and E. Witten, Nucl. Phys. B289, 589 (1987).

[9] L. Dixon, D. Friedan, E. Martinec, and S. Shenker, Nucl. Phys. B282, 13 (1987) S. Hamidi and C. Vafa, ibid. B279, 465 (1987).

[10] G. Pradisi and A. Sagnotti, Phys. Lett. B 216, 59 (1989); E. G. Gimon and J. Polchinski, Phys. Rev. D 54, 1667 (1996).

[11] E. G. Gimon and C. V. Johnson, Nucl. Phys. B477, 715 (1996), A. Dabholkar and J. Park, ibid. B477, 701 (1996).

[12] M. Bianchi, G. Pradisi, and A. Sagnotti, Nucl. Phys. B376, 365 (1992).

[13] J. D. Blum and K. Intriligator, "Consistency Conditions for Branes at Orbifold Singularitites,', hep-th/9705030.

[14] D. Friedan, E. Martinec, and S. Shenker, Phys. Lett. 160B, 55 (1985); Nucl. Phys. B271, 93 (1986). 